\title{
BMJ Open Protocol for a diagnostic accuracy study to develop diagnosis algorithm for biliary atresia using MMP-7 (DIABA-7 study): a study recruiting from Chinese Biliary Atresia Collaborative Network
}

\author{
Jingying Jiang, ${ }^{1}$ Jiale Deng, ${ }^{2}$ Gong Chen, ${ }^{1}$ Rui Dong, ${ }^{1}$ Song Sun, ${ }^{1}$ Min Du, ${ }^{1}$ \\ Dake Yang, ${ }^{2}$ Zhuo Fang, ${ }^{2}$ Shan Zheng (D) , ${ }^{1}$ Chinese Biliary Atresia Collaborative \\ Network
}

To cite: Jiang J, Deng J, Chen G, et al. Protocol for a diagnostic accuracy study to develop diagnosis algorithm for biliary atresia using MMP-7 (DIABA-7 study): a study recruiting from Chinese Biliary Atresia Collaborative Network. BMJ Open 2021;11:e052328. doi:10.1136/ bmjopen-2021-052328

- Prepublication history for this paper is available online. To view these files, please visit the journal online (http://dx.doi. org/10.1136/bmjopen-2021 052328).

$\mathrm{JJ}$ and JD contributed equally.

JJ and JD are joint first authors.

Received 13 April 2021

Accepted 02 December 2021

Check for updates

(c) Author(s) (or their employer(s)) 2021. Re-use permitted under CC BY-NC. No commercial re-use. See rights and permissions. Published by BMJ.

For numbered affiliations see end of article.

\section{Correspondence to} Dr Shan Zheng; szheng@shmu.edu.cn and Dr Zhuo Fang; fang_zhuo@wuxidiagnostics. com

\section{ABSTRACT}

Introduction Biliary atresia is a severe liver disease in neonates, and the prognosis partially depends on the age at which infants undergo the Kasai procedure. Matrix metalloproteinase-7 (MMP-7) was confirmed to have significant value in the diagnosis of biliary atresia. However, so far, the reference range and its cut-off value for diagnosing biliary atresia have not been established yet.

Methods and analysis Dlagnosis Algorithm for Biliary Atresia (DIABA-7) is a prospective diagnostic test. Cholestatic infants and normal controls within 150 days of age are recruiting from the Chinese Biliary Atresia Collaborative Network. The serum samples and dried blood spot (DBS) samples are obtained to detect MMP7 concentrations using an ELISA kit. The reference standard is the intraoperative exploration and subsequent histological examination of liver biopsies. Lambda-MuSigma (LMS) method is used to calculate the normal range of serum MMP-7 of each age group. Receiver operating characteristics (ROC) curves are constructed to calculate the best cut-off point and area under the curve for the index test. The sensitivity, specificity, positive predictive value and negative predictive value are used to show the diagnostic accuracy. Pearson correlation coefficient test is applied to assess the correlation of serum MMP-7 and DBS MMP-7.

Ethics and dissemination This study was reviewed and approved by the Ethics Committee of Children's Hospital of Fudan University (Number 2020-296). Dissemination will be guided by investigators and patients. The aim is to publish the study results in a high-quality peer-reviewed journal and present the findings at international academic meetings.

Trial registration number ChiCTR2000032983.

\section{INTRODUCTION}

Biliary atresia (BA) is a severe liver disease in neonates, characterised by fibroinflammatory destruction of bile ducts. ${ }^{1}$ Kasai portoenterostomy has been the consensual strategy
Strengths and limitations of this study

- This is a multicenter study in China recruiting 1200 consecutive cholestatic infants and 154 normal controls, which will be one of the largest diagnostic tests for biliary atresia (BA).

- The reference range and cut-off value for diagnosing BA of MMP-7 will be established so that to further standardise and popularise its clinical application.

- Diagnostic model based on serum biomarkers together with other parameters will help in the precise diagnosis for BA.

- External validation of the diagnostic cut-off value will be needed in the setting and population that the diagnosis algorithm will be used.

for BA since $1959 .{ }^{23}$ The age at which infants with BA undergo the Kasai procedure is a good clinical predictor of outcome, for those who undergo surgery within 60-90days of age achieve a better prognosis. ${ }^{4-7}$ However, prompt treatment relies on early diagnosis. So far, it remains difficult to achieve perfect accuracy with either liver function test or grayscale ultrasound, which are most widely used in clinical practice. ${ }^{8}$

Matrix metalloproteinase-7 (MMP-7) plays an important role in extracellular matrix remodelling, which is closely related to the progression of liver fibrosis. ${ }^{9}{ }^{10}$ In 2017, it was first reported by Bezerra et al that MMP-7 had a promising diagnostic value for BA, with an area under the curve (AUC) of 0.97 , and the sensitivity and specificity were $97 \%$ and $91 \%$, respectively. ${ }^{11}$ In 2018, Yang et alfrom the same group further conducted a clinical diagnostic test and found that the AUC for diagnosing BA was 0.99 at the cut-off value of $52.85 \mathrm{ng} /$ $\mathrm{mL}$, and the sensitivity, specificity and positive 
predictive value (PPV) were 98.67\%, 95\% and 98.28\%, respectively. ${ }^{12}$ Afterwards, groups from Taiwan and Shanghai also verified the diagnostic accuracy of serum MMP-7, and the sensitivity was $97.3 \%$ and $95.19 \%$, and the specificity was $83.2 \%$ and $93.07 \%$, respectively. ${ }^{1314}$ However, the cut-off value varied, $10.37 \mathrm{ng} / \mathrm{mL}$ for the study from Shanghai, while only $1.43 \mathrm{ng} / \mathrm{mL}$ for that from Taiwan.

All the studies mentioned above highly confirmed the significant value of serum MMP-7 in the diagnosis of BA, whereas the differences in the cut-off value indicated that further research is warranted before its clinical application, to standardise the measurement of serum MMP-7 and confirm the reference range and also its cut-off value for diagnosing BA.

\section{METHODS AND ANALYSIS \\ Study design}

DIagnosis Algorithm for Biliary Atresia (DIABA-7) is a prospective diagnostic test. A study protocol for recruitment plan and detailed data collection method was designed and related medical professionals were trained before the study's start date.

\section{Primary objective}

To clarify the normal reference range of serum MMP-7 for each month age within 150 days after birth and the diagnostic cut-off value in diagnosing BA.

\section{Secondary objectives}

1. To analyse the value of serum MMP-7 in the differential diagnosis of BA in each age group within 150 days, especially in the neonatal group.

2. To develop a convenient and simple-operated method using dried blood spot (DBS) to measure MMP-7 concentration, which can be delivered at room temperature, and to explore its feasibility in screening and diagnosing BA.

3. To establish an accurate multivariate diagnostic model of BA using the deep machine learning model based on serum biomarkers together with other parameters.

\section{Setting}

DIABA-7 is recruiting from Chinese Biliary Atresia Collaborative Network, including paediatric surgery or gastroenterology outpatient clinics and inpatient departments in the tertiary hospitals in China including Children's Hospital of Fudan University, Children's Hospital of Fudan University Xiamen Branch, HaiNan Children's Hospital, Children's Hospital of Shanxi, Children's Hospital of Nanjing Medical University, Shengjing Hospital of China Medical University, Hunan Children's Hospital, etc. The multicentre recruitment will help to achieve the sample goal within the timeline and support the generalisability of the test.

\section{PARTICIPANT SELECTION}

\section{Inclusion criteria}

1. Cholestatic patients

A. Age: $\leq 150$ days, no gender limit.
B. Diagnostic criteria for cholestatic jaundice: serum total bilirubin $<85 \mu \mathrm{mol} / \mathrm{L}$, but serum direct bilirubin $\geq 17 \mu \mathrm{mol} / \mathrm{L}$ or serum total bilirubin $\geq 85 \mu \mathrm{mol} / \mathrm{L}$ but direct bilirubin accounts for more than $20 \%$.

C. Gestational age $\geq 28$ weeks, birth weight $\geq 1000 \mathrm{~g}$.

D. Sign the informed consent form.

2. Normal controls

A. Age: $\leq 150$ days old, no gender limit.

B. Gestational age $\geq 28$ weeks, birth weight $\geq 1000 \mathrm{~g}$.

C. No manifestations of cholestasis or other known hepatobiliary diseases.

D. Sign the informed consent form.

\section{Exclusion criteria}

A. A history of severe hypoxia at birth, Apgar score $<5$ points.

B. A history of allergic diseases, inflammatory bowel disease and infectious diseases.

C. Complicated with other serious congenital malformations and immune deficiencies such as neurological, cardiovascular, pulmonary, endocrine and renal disorders that would interfere with the conduct and results of the study.

\section{INDEX TEST}

\section{Sample collection}

Blood samples of the cholestatic patients are obtained along with patients' routine laboratory tests at the outpatient clinics or at the admission to the inpatient unit.

Blood samples of the normal controls are obtained along with patients' routine laboratory tests at the admission to the inpatient unit from patients admitted for reasons other than hepatobiliary diseases.

The blood sample is centrifuged at $3000 \mathrm{rpm}$ for $10 \mathrm{~min}$ to get the serum, and then the serum samples are stored at $-80^{\circ} \mathrm{C}$ before measurement.

At the same time, a volume of $10 \mu \mathrm{L}$ blood sample is obtained from the heel tip to a DBS patented by WuXi Diagnostics. ${ }^{15}$ After fully drying, it is stored at $-20^{\circ} \mathrm{C}$ before measurement.

\section{Serum MMP-7 concentration measurement}

Serum MMP-7 concentration is measured using an enzyme-linked immunosorbent assay (ELISA, R\&D, DMP700 and WuXi Diagnostics' Self-developed ELISA kit). Considering the measurement range of the kit, the serum samples of cholestatic patients are diluted 10 to 30 times. All the measurements are performed by WuXi Diagnostics who are blinded to other test results. Each sample is provided with three technical replicates in each assay, and the mean is recorded.

\section{MMP-7 concentration measurement for DBS}

Add $150 \mu \mathrm{l}$ Calibrator Diluent (RD6-28) of the ELISA kit (R\&D, DMP700) into the DBS tower, and then soak, shake (Thermo-shaker BE-9008) for 1 hour. Thereafter, $50 \mu \mathrm{l}$ of the solvent extraction is loaded to detect the concentration of MMP7 on DBS using enzyme-linked 
immunosorbent assay (ELISA, R\&D, DMP700). Each sample is provided with two technical replicates, and the mean is recorded.

\section{Serum IL-33 concentration measurement}

Serum IL-33 concentration is measured using enzymelinked immunosorbent assay (ELISA, R\&D, D3300B). Each sample is provided with three technical replicates, and the mean is recorded.

\section{Serum bile acid profile measurement}

A liquid chromatography-tandem mass spectrometry system (self-developed kit by WuXi Diagnostics) is used to detect the concentration of each bile acid in human serum including cholic acid (CA), glycocholic acid, taurocholic acid, chenodeoxycholic acid (CDCA), glycochenodeoxycholic acid, taurochenodeoxycholic acid, deoxycholic acid, glycodeoxycholic acid, taurodeoxycholic acid, ursodeoxycholic acid (UDCA), glycoursodeoxycholic acid, tauroursodeoxycholic acid, lithocholic acid (LCA), glycolithocholic acid, taurolithocholic acid, taurohyocholic acid, hyodeoxycholic acid (HDCA), glycohyodeoxycholic acid, taurohyodeoxycholi acid, dehydrocholic acid.

\section{Reference test}

Intraoperative exploration and subsequent histological examination of liver biopsies are used to confirm the diagnosis of BA. If the gallbladders of some patients are seen to be completely atrophic, and the injection of contrast is hard to achieve, the baby will be diagnosed as BA at once. Otherwise, a cholangiography will be performed to further evaluate the anatomy of the intrahepatic bile ducts before a final diagnosis is made. Non-BA patients are confirmed by intraoperative cholangiography showing a patent biliary tree, percutaneous transhepatic biopsy excluding BA, genetic tests showing certain gene mutation or alleviation without surgical intervention during follow-up for at least 3 months. All the cases will be reviewed by the experts from the Chinese Biliary Atresia Collaborative Network before the final diagnosis is made.

\section{Study flow}

The study flow is depicted in figure 1 . Infants who meet the eligibility criteria will be consecutively recruited. After informed consent has been taken, all participants undergo the index tests and the reference test. All the index tests are performed by investigators from WuXi Diagnostics who are blinded to other test results. The reference test is performed afterward by the investigators (experts in paediatric surgery or gastroenterology) of each hospital. Though the investigators of each hospital have access to the results of index tests before making the final diagnosis, the biases are limited since the reference standards are definite and objective. Following that, information is extracted from the medical record including the demographic parameters, routine liver function test, sonographic features, index tests, reference standard (diagnosis of BA or Non-BA) and outcome after

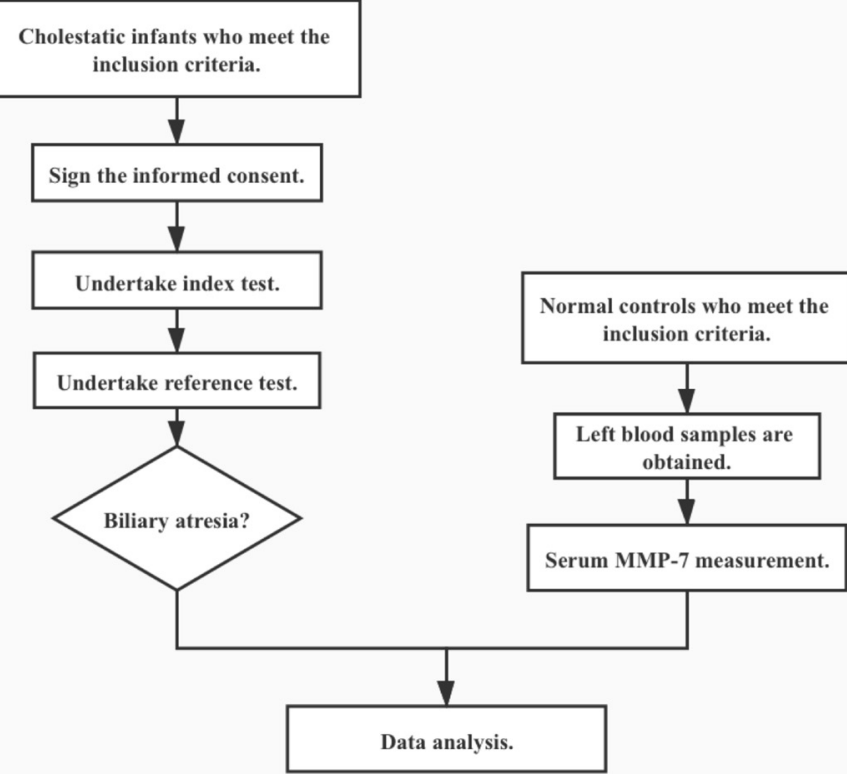

Figure 1 Flowchart of the study patient. MMP-7, matrix metalloproteinase-7.

treatment. A summary of the data variables collected in the DIABA-7 study is presented in table 1.

\section{Data management}

Data are collected at the time of measurement and from the medical record. Several steps have been taken to help ensure high-quality data collection. First, all DIABA-7 study investigators undergo standardised training before starting the study, including the sample collecting and storing, case report form filling. Besides, the electronic input is consistent with the content of the paper form. The data entry interface is established through Microsoft Access 2010 and uploaded to a central server on a weekly basis. Paper forms will also be available on the field as a backup.

\section{Sample size}

1. To clarify the normal reference range of serum MMP-7 and the diagnostic cut-off value in diagnosing BA, we set up the specificity of the test for distinguishing normal for case is $90 \%$, using the formula: $\mathrm{n}=\frac{\left[\mathrm{Z}_{1}-\alpha / 2\right]^{2} \mathrm{P}(1-\hat{\mathrm{P}})}{\mathrm{d}^{2}}$ , where $\hat{P}=0.9, \alpha=0.05, d=0.05 . n=139$.

2. To establish the correlation between serum MMP-7 and DBS MMP-7, 150 cholestatic participants will be needed with a two-tailed $\alpha$ of 0.05 and $\beta$ of 0.1 , for achieving correlation coefficient $r$ of 0.85 .

3. To prove the efficiency of BA artificial intelligence (AI) multivariate diagnostic model is better than the single biomarker, 581 samples will be needed for each group, by comparing the AUC. Due to our group's preliminary results and other literature, the hypothesis is: $\mathrm{H}_{0}: A U C_{A I}=A U C_{\text {singlebiomarker }}$ $\mathrm{H}_{1}: A U C_{A I}>A U C_{\text {singlebiomarker }}$ where $A U C_{A I}=0.956, A U C_{\text {singlebiomarker }}=0.92$.

Due to the statistical estimating above, the largest sample size request is 545 sample each group. 
Table 1 Case report form

\section{Patient ID: Number}

Baseline characteristics

Date of birth

Sex

Gestational age

Birth weight

Congenital anomalies

Preoperative biochemistry

Date of preoperative test

Platelet

Total bilirubin

Direct bilirubin

Alanine aminotransferase

Aspartate aminotransferase

Gamma-glutamyl

transpeptidase

Total bile acid

Albumin

Prothrombin time

Activated partial

thromboplastin time

International normalised ratio

Cholic acid

Glycocholic acid

Taurocholic acid

Chenodeoxycholic acid

Glycochenodeoxycholic acid

Taurochenodeoxycholic acid

Deoxycholic acid

Glycodeoxycholic acid

Taurodeoxycholic acid

Ursodeoxycholic acid

Glycoursodeoxycholic acid

Tauroursodeoxycholic acid

Lithocholic acid

Glycolithocholic acid

Taurolithocholic acid

Taurohyocholic acid

Hyodeoxycholic acid

Glycohyodeoxycholic acid

Taurohyodeoxycholi acid

Dehydrocholic acid

Matrix metalloproteinase 7

Interleukin 33

Preoperative sonographic

features

Date of ultrasound
Table 1 Continued

\begin{tabular}{ll}
\hline Patient ID: Number & \\
\hline Triangular cord sign & $\leftarrow$ Yes, _No \\
Gallbladder & $\leftarrow$ (size) \\
& $\leftarrow$ No \\
Common bile duct & $\leftarrow$ Yes, \\
& $\leftarrow$ No \\
Hepatic artery & \\
Liver stiffness measurement & \\
Final diagnosis & \\
Surgical exploration & \\
Liver biopsy \\
Gene test \\
Other
\end{tabular}

In conclusion, with the $5 \%$ data loss rate and the $5 \%$ screening fail rate, 1200 cases of cholestatic jaundice are proposed to be included, including 600 children with BA after intraoperative cholangiography (surgical gold standard) confirmation and 600 cholestatic children with other definite diagnosis than BA as well as 154 nonhepatobiliary disease controls to establish a reference interval.

\section{Data analysis}

Baseline characteristics of patients in the BA and non-BA groups are demonstrated using frequency distributions and descriptive statistics. Gender is described by n (\%). Continuous variables are expressed as mean $\pm \mathrm{SD}$, median and quartiles $(\mathrm{Q} 1, \mathrm{Q} 3)$. In the univariate analysis, the $\chi^{2}$ test will be conducted for gender, while Mann-Whitney $\mathrm{U}$ test will be performed for all continuous variables failing the Shapiro-Wilk W test for normality, otherwise, the student $t$ test will be used. Multivariate logistic regression will be used to establish a diagnostic model based on several parameters.

Using the Lambda-Mu-Sigma (LMS) method, that is, using the median $\mathrm{M}(\mathrm{mu})$, the coefficient of variation $\mathrm{S}$ (sigma) and the Box-Cox conversion power L (lambda), which is required to convert the data to the normal distribution, the normal range of serum MMP-7 of each age group will be calculated.

Receiver operating characteristics (ROC) curves are constructed to calculate the best cut-off point and AUC for each index test. The sensitivity, specificity, PPV and negative predictive value are used to show the diagnostic accuracy.

Pearson correlation coefficient test will be applied to assess the correlation of serum MMP-7 and DBS MMP-7.

The least absolute shrinkage and selection operator regression method will be used to determine the optimal covariates to differentiate BA form non-BA. The patients will be divided into training set (70\% data) and validating set (30\% data) by random sampling function in $\mathrm{R}$ 
software. The covariates picked will be used to build the multivariable logistic regression model. The accuracy of the regression model will be assessed by the discrimination ability in the training set and the validating set.

Statistically significant difference was defined as a $p$ value less than 0.05 . All data analyses are performed using R software (3.6.3, Vienna Australia).

\section{Patient and public involvement}

Patients and their guardians are not involved in the study design and are not consulted to develop patient-relevant diagnoses and outcomes or interpret the results. Patients are not invited to contribute to the writing or editing of this document for readability or accuracy. We intend to disseminate the main results to the families of the trial participants and will pursue patient and public involvement in the development of an appropriate method to disseminate this information.

\section{ETHICS AND DISSEMINATION \\ Ethics}

This study was reviewed and approved by the Ethics Committee of Children's Hospital of Fudan University (number 2020-296). The study will be performed in compliance with the Declaration of Helsinki and other relevant regulations.

The investigator has the responsibility to comprehensively introduce the purpose, procedures and possible risks of this study. An informed consent form must be given to each subject and be signed before recruitment.

The personal information will be kept confidential. Similarly, deidentified data are used for statistical evaluation. Only the investigators can identify the subject's name and other personal information by initials.

\section{Disseminations}

Dissemination will be guided by investigators and patients. The aim is to publish the study results in a high-quality peer-reviewed journal and present the findings at international academic meetings.

\section{DISCUSSION}

Experts from all over the world have made great contributions to exploring early and non-invasive diagnostic methods of BA during the past decades.

Stool colour cards can help identify the alcoholic stool in the early neonatal period, and the sensitivity for screening BA is around $76 \%-90 \%$ and the specificity is nearly $100 \% \cdot{ }^{16-20}$ In 2016, we launched the mobile phone application of stool colour card to simply and effectively differentiate abnormal stool from a normal one. Subsequently, a nomogram model based on five variables (gender, weight, direct bilirubin, alkaline phosphatase, glutamyltranspeptidase) was established based on big data with the sensitivity and specificity of $85.7 \%$ and $80.3 \%$, respectively, ${ }^{21}$ which helps the general practitioner to predict the risk of BA in cholestatic infants.

The age of Kasai operation in children with BA before and after stool colour card screening and nomogramassisted diagnosis (2014-2016 vs 2017-2019) in our hospital was statistically analysed. The results showed that the age of Kasai operation in the second 3 years $(62.68 \pm 19.60 \mathrm{~d})$ was earlier than that in the first 3 years (66.68 days \pm 24.89 days) $(\mathrm{p}>0.05)$.

Considering the convenience and accuracy of MMP-7 in diagnosing BA, a standardised diagnosis algorithm should be established to further improve the age of Kasai operation.

\section{Study process}

Seven tertiary hospitals in China are now open for recruitment. These centres are Children's Hospital of Fudan University, Children's Hospital of Fudan University Xiamen Branch, HaiNan Children's Hospital, Children's Hospital of Shanxi, Children's Hospital of Nanjing Medical University, Shengjing Hospital of China Medical University, Hunan Children's Hospital. The first participant was recruited in August 2020 and the study is due to finish recruiting in August 2022. We are currently in the subject recruitment. Other clinical centres for BA are still welcome to join in the Chinese Biliary Atresia Collaborative Network and the subject recruitment.

\section{Author affiliations}

${ }^{1}$ Department of Pediatric Surgery, Children's Hospital of Fudan University, Shanghai, China

${ }^{2}$ Department of Data \& Analytics, WuXi Diagnostics, Shanghai, China

Acknowledgements We would like to thank professor Weili Yan from Department of Clinical Epidemiology, Children's Hospital of Fudan University for the help in study design and statistical analyses. We are grateful to all the doctors and nurses in the medical centres involved for their contributions in the collection of the questionnaires and process of the study.

Collaborators Children's Hospital of Fudan University, Children's Hospital of Fudan University Xiamen Branch, HaiNan Children's Hospital, Children's Hospital of Shanxi, Children's Hospital of Nanjing Medical University, Shengjing Hospital of China Medical University, Hunan Children's Hospital , Children's Hospital of Xuzhou, Affliated Hospital of Zunyi Medical University, Chengdu Women's and Children's Central Hospital

Contributors SZ, ZF, JJ, JD conceptualised and designed the study, drafted the initial manuscript and reviewed and revised the manuscript. JJ, JD, DY, MD designed the standard questionnaire and the data collection instruments, collected data and reviewed and revised the manuscript. SZ, ZF, GC, RD, SS conceptualised and designed the study, coordinated and supervised data collection, and critically reviewed the manuscript for important intellectual content. All authors approved the final manuscript as submitted and agree to be accountable for all aspects of the work.

Funding This study received financial support from Shanghai Key Disciplines (number 2017ZZ02022), Shanghai Municipal Key Clinical Specialty (number shslczdzk05703), National Natural Science Foundation of China (number 81770519 , number 81771633 , number 81873545 and number 81974059), The Science Foundation of Shanghai (number 18411969100 and number 19ZR1406600), Children's National Medical Center (number EK1125180104, number EKYY20180204, EK112520180211 and number EK112520180310), and Shanghai Pujiang Program.

Competing interests None declared.

Patient and public involvement Patients and/or the public were not involved in the design, or conduct, or reporting, or dissemination plans of this research. 
Patient consent for publication Consent obtained from parent(s)/guardian(s).

Provenance and peer review Not commissioned; externally peer reviewed.

Open access This is an open access article distributed in accordance with the Creative Commons Attribution Non Commercial (CC BY-NC 4.0) license, which permits others to distribute, remix, adapt, build upon this work non-commercially, and license their derivative works on different terms, provided the original work is properly cited, appropriate credit is given, any changes made indicated, and the use is non-commercial. See: http://creativecommons.org/licenses/by-nc/4.0/.

ORCID iD

Shan Zheng http://orcid.org/0000-0002-9712-4573

\section{REFERENCES}

1 Alagille D. Extrahepatic biliary atresia. Hepatology 1984;4:7S-10.

2 Hartley JL, Davenport M, Kelly DA. Biliary atresia. Lancet 2009;374:1704-13.

3 Davenport M. Biliary atresia: clinical aspects. Semin Pediatr Surg 2012;21:175-84.

4 Feldman AG, Mack CL. Biliary atresia: clinical lessons learned. $J$ Pediatr Gastroenterol Nutr 2015;61:167-75.

5 Kelay A, Davenport M. Long-Term outlook in biliary atresia. Semin Pediatr Surg 2017;26:295-300.

6 Lopez RN, Ooi CY, Krishnan U, Early KU. Early and peri-operative prognostic indicators in infants undergoing hepatic Portoenterostomy for biliary atresia: a review. Curr Gastroenterol Rep 2017:19:16.

7 Song Z, Dong R, Shen Z, et al. Surgical outcome and etiologic heterogeneity of infants with biliary atresia who received Kasai operation less than 60 days after birth: a retrospective study. Medicine 2017;96:e7267.

8 He J-P, Hao Y, Wang X-L, et al. Comparison of different noninvasive diagnostic methods for biliary atresia: a meta-analysis. World $J$ Pediatr 2016;12:35-43.

9 Berg G, Barchuk M, Miksztowicz V. Behavior of metalloproteinases in adipose tissue, liver and arterial wall: an update of extracellular matrix remodeling. Cells 2019;8. doi:10.3390/cells8020158. [Epub ahead of print: 1402 2019].

10 Geervliet E, Bansal R. Matrix metalloproteinases as potential biomarkers and therapeutic targets in liver diseases. Cells 2020;9:1212.

11 Lertudomphonwanit C, Mourya R, Fei L, et al. Large-Scale proteomics identifies MMP-7 as a sentinel of epithelial injury and of biliary atresia. Sci Trans/ Med 2017;9. doi:10.1126/scitranslmed. aan8462. [Epub ahead of print: 22 Nov 2017].

12 Yang L, Zhou Y, Xu P-P, et al. Diagnostic accuracy of serum matrix metalloproteinase-7 for biliary atresia. Hepatology 2018;68:2069-77.

13 Jiang J, Wang J, Shen Z, et al. Serum MMP-7 in the diagnosis of biliary atresia. Pediatrics 2019;144. doi:10.1542/peds.2019-0902. [Epub ahead of print: 1110 2019].

$14 \mathrm{Wu}$ J-F, Jeng Y-M, Chen H-L, et al. Quantification of serum matrix metallopeptide 7 levels may assist in the diagnosis and predict the outcome for patients with biliary atresia. J Pediatr 2019;208:30-7.

15 Hang H, Zhang Y, Zhang XH. Dried blood spot quantitative collection device and method. Shanghai: CN111474257A,2020-07-31, 2020.

16 Chen S-M, Chang M-H, Du J-C, et al. Screening for biliary atresia by infant stool color card in Taiwan. Pediatrics 2006;117:1147-54.

$17 \mathrm{H}$ siao $\mathrm{C}-\mathrm{H}$, Chang $\mathrm{M}-\mathrm{H}$, Chen $\mathrm{H}-\mathrm{L}$, et al. Universal screening for biliary atresia using an infant stool color card in Taiwan. Hepatology 2008;47:1233-40.

18 Gu Y-H, Yokoyama K, Mizuta K, et al. Stool color card screening for early detection of biliary atresia and long-term native liver survival: a 19-year cohort study in Japan. J Pediatr 2015;166:897-902.

19 Kong Y-Y, Zhao J-Q, Wang J, et al. Modified stool color card with digital images was efficient and feasible for early detection of biliary atresia-a pilot study in Beijing, China. World $\mathrm{J}$ Pediatr 2016;12:415-20.

20 Woolfson JP, Schreiber RA, Butler AE, et al. Province-wide biliary atresia home screening program in British Columbia: evaluation of first 2 years. J Pediatr Gastroenterol Nutr 2018;66:845-9. doi:10.1097/MPG.0000000000001950

21 Dong R, Jiang J, Zhang S, et al. Development and validation of novel diagnostic models for biliary atresia in a large cohort of Chinese patients. EBioMedicine 2018;34:223-30. 